\title{
Computational Analysis of Particulate Flow in Expansion Channel
}

\author{
Nor Azwadi Che Sidik and Leila Jahanshaloo \\ Department of Thermo-Fluid, Faculty of Mechanical Engineering, \\ University Technology Malaysia, 81310 UTM Skudai, Johor, Malaysia
}

Received 2011-05-28, Revised 2012-07-27; Accepted 2013-05-07

\begin{abstract}
Computational prediction of fluid-solid particle interaction in an expansion horizontal channel with wide range of Reynolds numbers. Lagrangian-Lagrangian numerical technique to predict the movement of solid particle. The method is based on mesocale scheme of lattice Boltzmann method for prediction of fluid dynamics and second Newton's law for the dynamics of solid particles. The flow behaviour at the downstream of the expansion channel is critically dependence on the Reynolds number of the flow. The removal percentage of the contaminant critically dependence on the flow structure donwstream of the expansion channel. The strength of recirculation region plays significant role due to the step in the cavity.
\end{abstract}

Keywords: Lagrangian Scheme, Lattice Boltzmann, Second Newton's Law, Expansion Pipe, Reynolds Number, Particulate Flow

\section{INTRODUCTION}

Knowledge of the particle motion in fluid flow is central in many natural and industrial applications. Examples are sediment grains in rivers, cloud particles in the atmosphere, fluidized beds and fluid-solid cyclone separators. Comprehending the major mean particlefluid flow phenomena, such as fluid-solid interaction and particles removal or mixing, is crucial for the environmental management and design and operation of such engineering equipment. A major limitation, in the problem of particle motion in fluid flow, is the insufficient information about the interaction between the fluid dynamics parts and solid particle flows. Although there are few fundamental experimental investigations which dictate that formation of vortex enhances the mixing process and strong vortex helps in the process of contaminant removal, more detailed phenomena such as the interaction between the vortex formation and the percentage of contaminant removal and flow parameters on the particles dynamics are still not fully understood.

Hydrodynamics cleaning of components, parts and pipelines has become widely accepted as an alternative method of cleaning process. However, other problems arise when the presence of distinct cavities and steps due to poorly fitted components or connections in the pipelines. These contributed to the accumulation of contaminants and cleaning of these can lead to quite difficult problems. Formation of vortices in cavities or steps can either enhance or prevent the removal of contaminant and depend on various flow parameters and characteristic of the contaminant itself. In order to highlight the relevance of the presence work, a brief review of the major efforts describing the cleaning process is presented.

Till present day, only a few papers have been reported to experimentally investigate this kind of

Corresponding Author: Nor Azwadi Che Sidik, Department of Thermo-Fluid, Faculty of Mechanical Engineering,

University Technology Malaysia, 81310 UTM Skudai, Johor, Malaysia

Tel: 07-5534628 Fax: +60-7-5566159 
multiphase flow. Due to these reasons, the problem relating to this case still not fully understood. The first experimental work on the fluid-particle interaction was reported by Saffman and Turner (1956). Their major objective was to identify the possible correlation between the collision rate among the same size of droplets and turbulent intensity in atmospheric clouds. Not much improvement has been made till the works by Abrahamson (1975) who extended the formulation for investigation at high turbulent intensity and without external force. Recently, Tsorng et al. (2006) experimentally investigated various external effects on the trajectories of small particle in shear driven cavity flow. Other experimental works are Adrian (1991); Ushijima and Tanaka (1996); Ide and Ghil (1997); Han et al. (1999); Hu (2002); Liao (2002) and Matas et al. (2004).

However, as remarked by Jie et al. (2008), most of the experimental studies have been limited to relatively simple cases and simple geometries. In real engineering applications, for example, cyclone separator, the chamber is specifically designed so that the vortex flow will induce high pressure gradient and the fluid-solid interaction process in such system is practically unpredictable.

On the other side, numerical research on fluidsolid particles begins immediately after the introduction of computer as a new tool for research activities (Shigeru and Saito, 1970). Since then, we can see vast of numerical researches on the subject, for example Kosinski et al. (2009); Ilea et al. (2008); Sommerfeld (2001) and Sidik and Attarzadeh (2012).

Recently, lattice Boltzmann method has evolved as a new approach to predict the fluid flow problem (Azwadi and Idris, 2010). However, the application of lattice Boltzmann method to fluid solid interaction has not attained great success due to the severe numerical instability caused by the different scale condition (Frisch et al., 1986; Azmir and Azwadi, 2009; Wolf-Gladrow, 2000).

There are two major objectives of the present article. The first is to extend the method for the integration between the meso-scale of LBM and the macro-scale of physical condition. The second objective is to shed the light on the fluid-solid interaction phenomenon that take place in an expansion pipe channel, which has not fully covered in the literature. The numerical scheme is firstly described and then the computational model results are presented and discussed. The parameters of major concern herein are the vortex structure, rate of contaminant removal and the percentage of removal at the steady state.

\section{MATERIALS AND METHODS}

\subsection{Numerical Scheme}

Recently, there are a lot of researches applying the Lattice Boltzmann Method (LBM) to study various types of fluid flow problem (Azwadi et al., 2010; Azwadi and Ibrahim, 2010; Hall and Fraser, 2006; Azwadi and Syahrullail, 2009). The LBM originate from the kinetic Boltzmann equation derived by Ludwig Boltzmann (1844-1906) in 1988. LBM has emerged as a versatile numerical method for simulating various types of fluid flow problem including turbulent (Jonas et al., 2005), multiphase (Alapati et al., 2008), magneto hydrodynamics (Breyiannis and Valougeorgis, 2004), flow in porous media (Guo and Zhao, 2002), microchannel flow (Zhang et al., 2005).

To predict the fluid flow, we consider the lattice Boltzmann Equation (1):

$$
\frac{\partial \mathrm{f}_{\mathrm{i}}}{\partial \mathrm{t}}+\mathbf{c}_{\mathrm{i}} \frac{\partial \mathrm{f}}{\partial \mathbf{x}}=-\frac{1}{\tau}\left(\mathrm{f}_{\mathrm{i}}-\mathrm{f}_{\mathrm{i}}^{\mathrm{eq}}\right)
$$

where, $f_{i}^{e q}$ is the equilibrium distribution function. is the lattice velocity and $\mathrm{i}$ is the lattice direction, $\mathrm{t}$ is the relaxation times of the particle distribution functions, respectively. The macroscopic variables such as the density and fluid velocity can be computed in terms of the particle distribution functions as in Equation (2):

$$
\begin{aligned}
& \rho=\sum_{\mathrm{i}} \mathrm{f}_{\mathrm{i}}^{\mathrm{eq}} \\
& \rho \mathbf{u}=\sum_{\mathrm{i}} \mathbf{c}_{\mathrm{i}} \mathrm{f}_{\mathrm{i}}^{\mathrm{eq}}
\end{aligned}
$$

The equilibrium distribution functions are given as in Equation (3):

$$
\mathrm{f}_{\mathrm{i}}^{\mathrm{eq}}=\rho \omega_{\mathrm{i}}\left[1+3 \mathbf{c}_{\mathrm{i}} \cdot \mathbf{u}+4.5\left(\mathbf{c}_{\mathrm{i}} \cdot \mathbf{u}\right)^{2}-1.5 \mathbf{u}^{2}\right]
$$

where, $\omega$ is the weight function (Mussa et al., 2011).

\subsection{Problem Physics}

The physical domain of the problem is represented in Fig. 1. The inlet center velocity was varied to give the Reynolds number ranges from 100-600. A swamp of solid particle was located just downstream of the expansion step as in Fig. 1. 


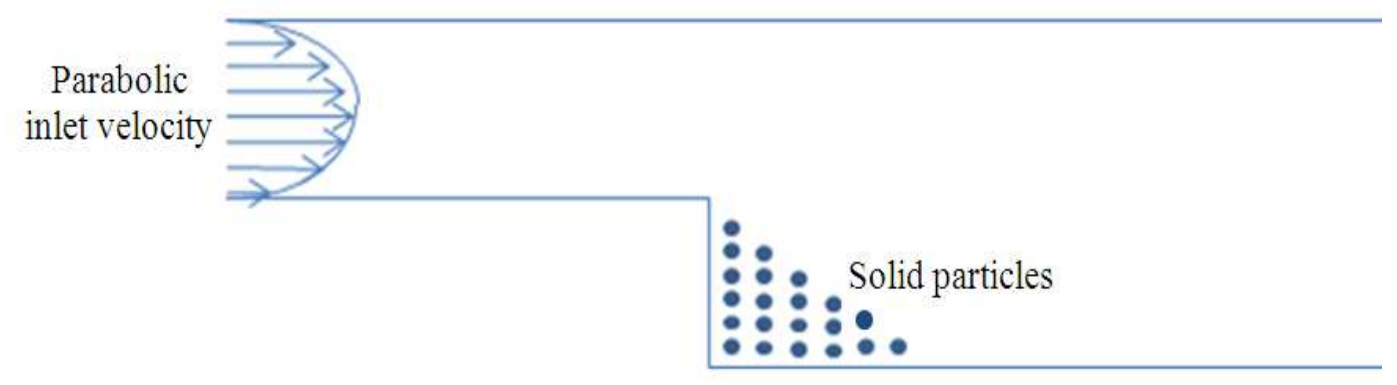

Fig. 1. Removal of contaminant in expansion pipe

In the present investigation, we only consider one way interaction which the presence of solid particles gives no effect to the fluid flow. The equation of motion for solid particles is written as in Equation (4):

$\mathrm{m}_{\mathrm{p}} \frac{\mathrm{d} \mathbf{v}_{\mathrm{p}}}{\mathrm{dt}}=\mathbf{f}_{\mathrm{p}}$

The drag force in the above equation can be expressed as Equation (5 and 6):

$\mathbf{f}_{\mathrm{p}}=\mathrm{C}_{\mathrm{D}} \mathrm{A}_{\mathrm{p}} \rho \frac{\left(\mathbf{u}-\mathbf{v}_{\mathrm{p}}\right)\left|\mathbf{u}-\mathbf{v}_{\mathrm{p}}\right|}{2}$

Where:

$\mathrm{C}_{\mathrm{D}}=\frac{24}{\operatorname{Re}_{\mathrm{p}}}\left(1+\frac{1}{6} \operatorname{Re}_{\mathrm{p}}^{2 / 3}\right)$

here $\mathrm{Re}_{\mathrm{p}}$ is the Reynolds number of solid particle.

In the present analysis, since we are coupling the macroscopic unit for solid particle and mesoscopic unit for lattice Boltzmann formulation, it is crucial to understand the relationship between these two different scales of units. For the present system, the Reynolds number of the particle must be set the same both in lattice Boltzmann formulation and actual physical flow as in Equation (7):

$\operatorname{Re}_{p}=\frac{u_{L} d_{L}}{v_{L}}=\frac{u_{R} d_{R}}{v_{R}}$

Here, the subscripts $\mathrm{L}$ and $\mathrm{R}$ denote the variables in lattice units and physical units, respectively. Hence, the actual time must be converted from lattice time to physical time as in Equation (8):

$$
t_{R}=\left(\frac{d_{R}}{d_{L}}\right)^{2}\left(\frac{d_{L}}{d_{R}}\right) t_{L}
$$

\section{RESULTS}

In the present analysis, the transient hydrodynamic removal of solid particles from in an expansion channel is presented. In the simulation, the Reynolds number was set up at three different values $(100,400$ and 600) which are based on the maximum inlet flow velocity and the channel height.

Figure 2-4 shows how the process of solid particle removal which initially trapped behind the step in horizontal pipe. As demonstrated by the figures, the highest rate of removal occurs on the early formation of vortex downstream the expansion step. Then, when the recirculation region is shaped, it trapped some of the particles until steady state is achieved.

Reynolds number also plays significant effect on the percentage of particle removal from the cavity. These are presented in Fig. 5, where the comparisons of removal percentage are made for three Reynolds numbers. Higher value of Reynolds number results in bigger vortex size downstream the expansion. This will drag the solid particles at the lower region nearer the walls and due to the inertia force, these particles are flushed to the downstream channel. However, even at the highest Reynolds number in the present study, the maximum percentage of removal is quite low which is around $55 \%$. 
Nor Azwadi Che Sidik and Leila Jahanshaloo / American Journal of Applied Sciences, 10 (4): 388-394, 2013

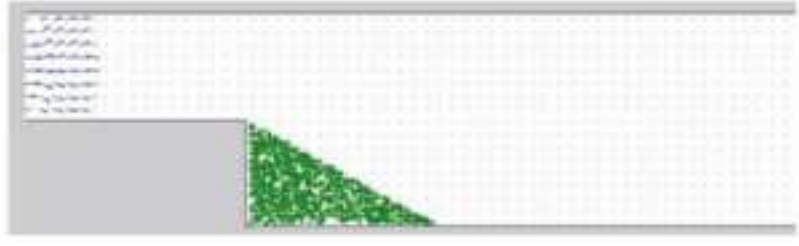

(a)

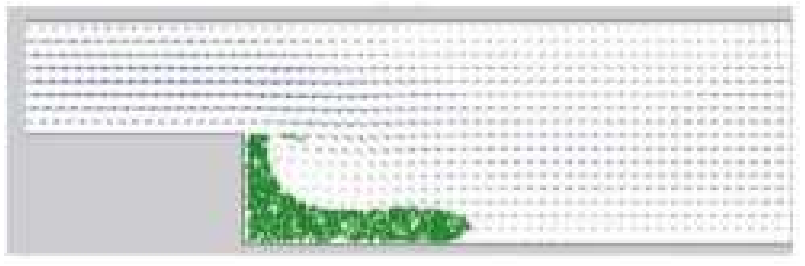

(c)

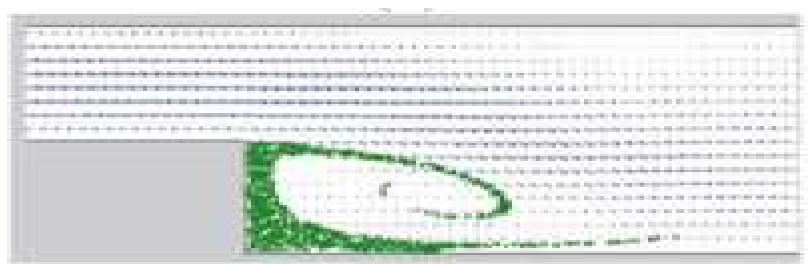

(e)

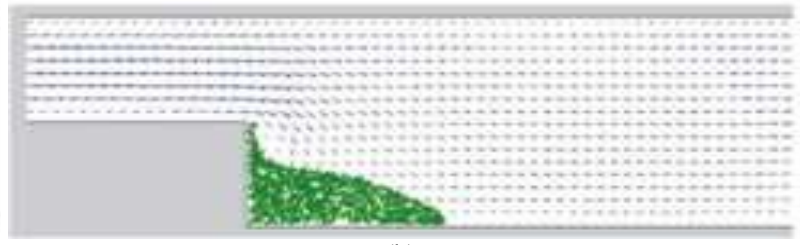

(b)

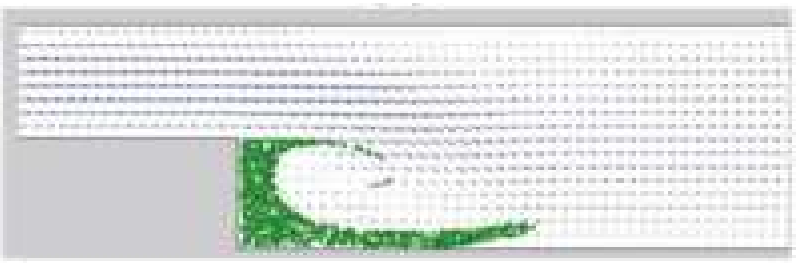

(d)

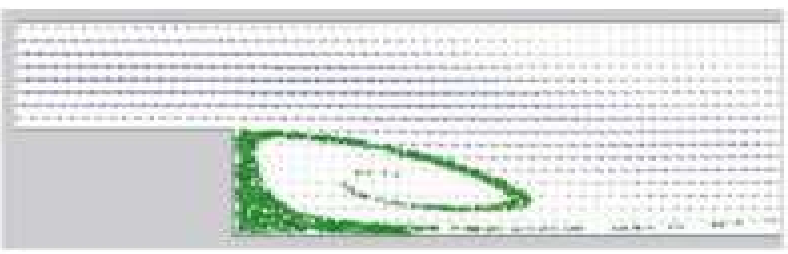

(f)

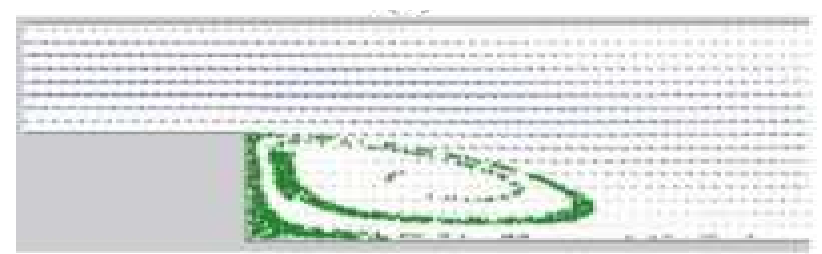

(g)

Fig. 2. Transient removal of contaminant in expansion pipe for $\mathrm{Re}=100$

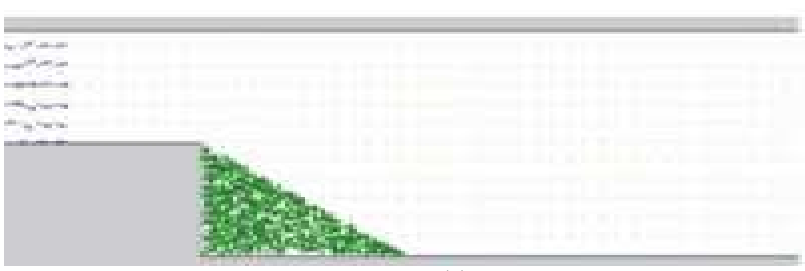

(a)

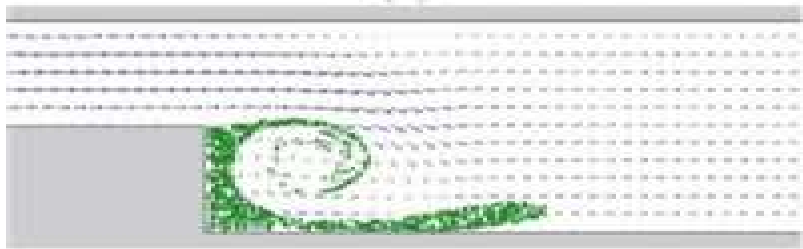

(c)

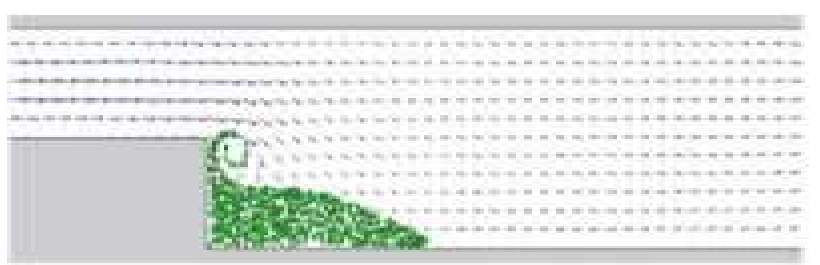

(b)

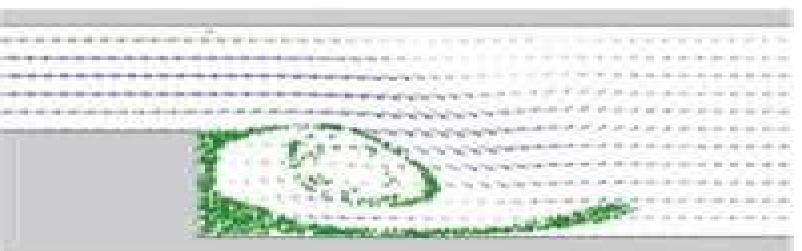

(d) 
Nor Azwadi Che Sidik and Leila Jahanshaloo / American Journal of Applied Sciences, 10 (4): 388-394, 2013

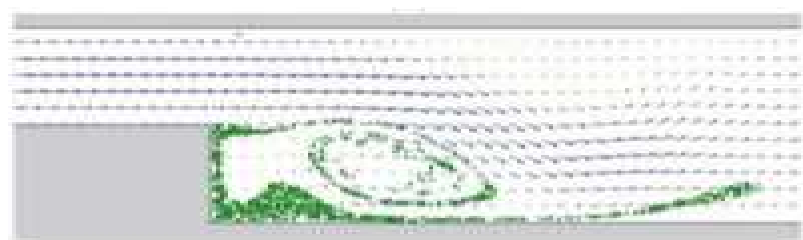

(e)

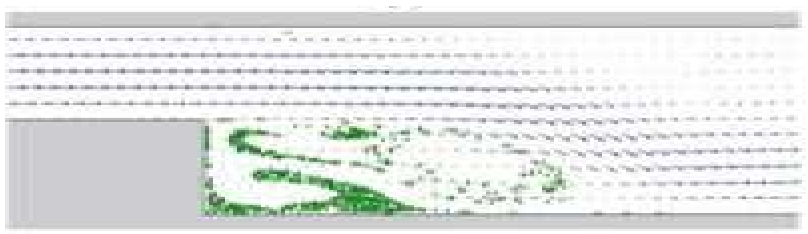

(g)

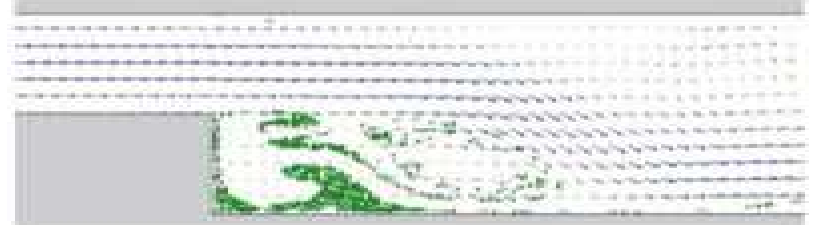

(f)

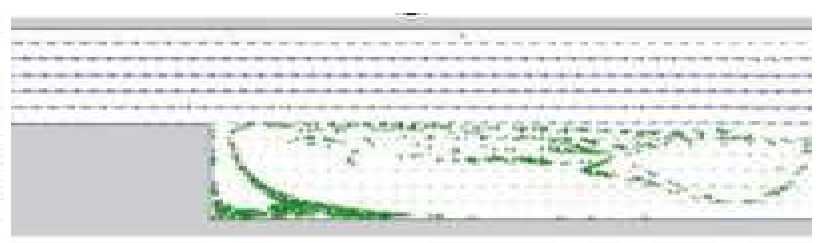

(h)

Fig. 3. Transient removal of contaminant in expansion pipe for $\mathrm{Re}=400$

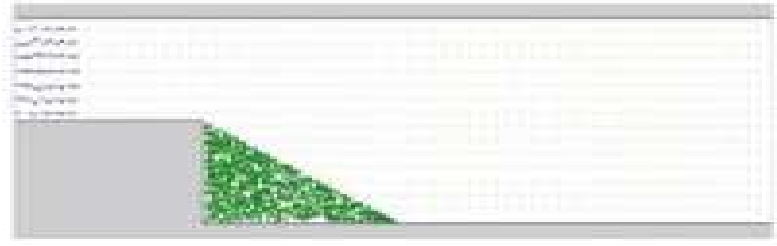

(a)

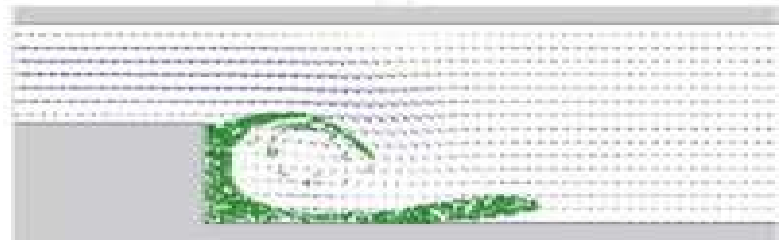

(c)

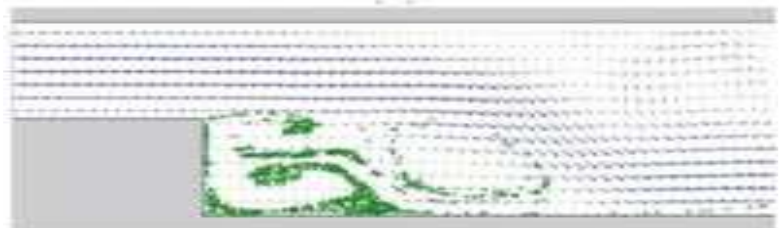

(e)

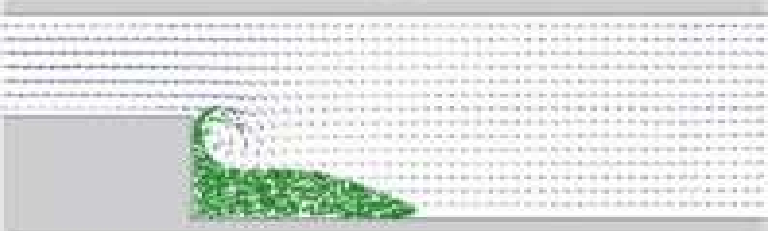

(b)

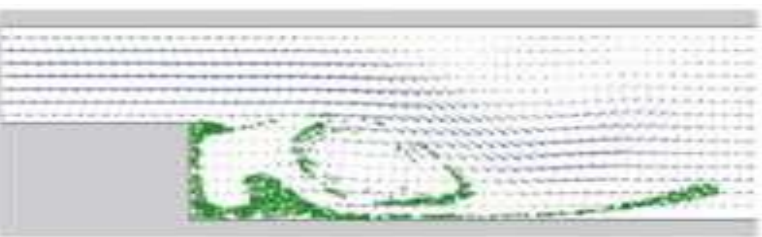

(d)

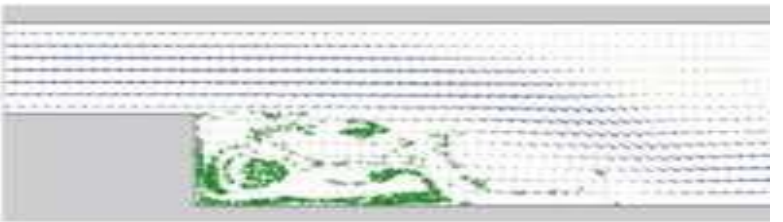

(f)

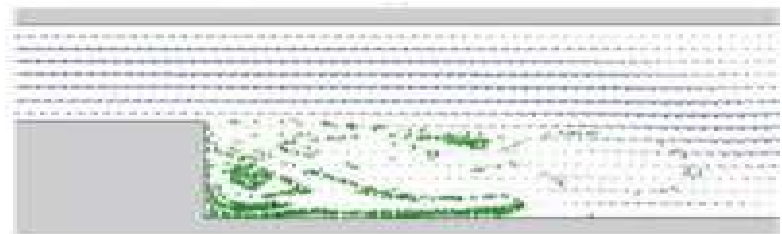

(g)

Fig. 4. Transient removal of contaminant in expansion pipe for $\mathrm{Re}=600$ 


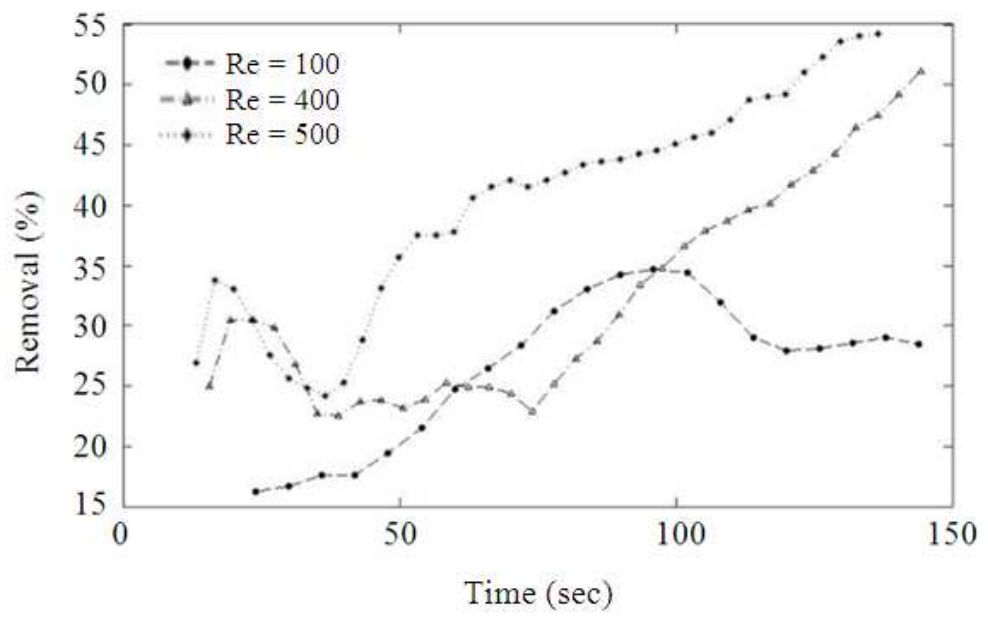

Fig. 5. Percentage of removal for three reynolds numbers

\section{DISCUSSION}

For the all cases, we can see at steady state condition, the remaining particles are still trapped near the walls downstream the expansion. These particles can be further removed by introducing the buoyancy force on the particles. This can be done by heating up the bottom wall to induce diffusion effect on the fluid as proposed by Chilukuri and Middleman (1983). Further discussion on this subject will be our next research topic.

\section{CONCLUSION}

Numerical computations of transient hydrodynamics of solid particles were performed using the LagrangianLagrangian scheme. The lattice Boltzmann method has been coupled with the second Newton's law to predict the dyanmics of solid particles.

The combination of scheme is applied to a twodimensional fluid-solid particle in expansion horizontal channel at three different Reynolds numbers. Results show that the proposed scheme method produce reasonably accurate results at low Reynolds numbers and stable results at medium Reynolds numbers. Extension to three-dimensional prediction of the geometry will be our next research topic.

\section{REFERENCES}

Abrahamson, J., 1975. Collision rates of small particles in a vigorously turbulent fluid. Chem. Eng. Sci., 30: 1371-1379. DOI: 10.1016/0009-2509(75)85067-6
Adrian, R.J., 1991. Particle-imaging techniques for experimental fluid mechanics. Ann. Rev. Fluid Mechan., 23: 261-304. DOI: 10.1146/annurev.fl.23.010191.001401

Alapati, S., S. Kang and Y.K. Suh, 2008. 3D Lattice boltzmann simulation of droplet formation in a cross-junction microchannel. Proceedings of the 3rd IASME/WSEAS International Conference on Continuum Mechanics, (CM'08), pp: 150-156.

Azmir, O.S. and C.S.N. Azwadi, 2009. UTOPIA finite different lattice Boltzmann method for simulation natural convection heat transfer from a heated concentric annulus cylinder. Eur. J. Scient. Res., 38: 63-71.

Azwadi, C.S.N. and M.S. Idris, 2010. Mesoscale numerical approach to predict macroscale fluid flow problems. J. Applied Sci., 10: 1511-1524. DOI: 10.3923/jas.2010.1511.1524

Azwadi, C.S.N. and N.I.N. Ibrahim, 2010. Lattice Boltzmann numerical scheme for the simulation of natural convection in an inclined square cavity. WSEAS Trans. Math., 9: 417-426.

Azwadi, C.S.N. and S. Syahrullail, 2009. A threedimension double-population thermal lattice BGK model for simulation of natural convection heat transfer in a cubic cavity. WSEAS Trans. Math., 8: 561-571.

Azwadi, C.S.N., M.Y.M. Fairus and S. Syahrullail, 2010. Virtual study of natural convection heat transfer in an inclined square cavity. J. Applied Sci., 10: 331336. DOI: $10.3923 /$ jas.2010.331.336 
Breyiannis, G. and D. Valougeorgis, 2004. Lattice kinetic simulations in three-dimensional magnetohydrodynamics. Phys. Rev., 69: 065702065705. DOI: 10.1103/PhysRevE.69.065702

Chilukuri, R. and S. Middleman, 1983. Circulation, diffusion and reaction within a liquid trapped in a cavity. Chem. Eng. Commun., 22: 127-138. DOI: 10.1080/00986448308940051

Frisch, U., B. Hasslacher and Y. Pomeau, 1986. Latticegas automata for the Navier-Stokes equation. Phys. Rev. Let., 56: 1505-1508. DOI: 10.1103/PhysRevLett.56.1505

Guo, Z. and T.S. Zhao, 2002. Lattice Boltzmann model for incompressible flows through porous media. Phys. Rev. E, 66: 036034-036042. DOI: 10.1103/PhysRevE.66.036304

Hall, R. and N.D. Fraser, 2006. Simulating acoustic propagation using a lattice boltzmann model of incompressible fluid flow. WSEAS Trans. Signal Process., 2: 876-881.

Han, M., C. Kim, M. Kim and S. Lee, 1999. Particle migration in tube flow of suspensions. J. Rheol., 43: 1157-1174. DOI: 10.1122/1.551019

$\mathrm{Hu}$, C.C., 2002. Analysis of motility for aquatic sperm in videomicroscopy. Ph.D. Thesis, National Taiwan University.

Ide, K. and M. Ghil, 1997. Extended Kalman filtering for vortex systems. Part 1: Methodology and point vortices. Dynam. Atmos. Oceans, 27: 301-332. DOI: 10.1016/S0377-0265(97)00016-X

Ilea, C.G., P. Kosinski and A.C. Hoffmann, 2008. Threedimensional simulation of a dust lifting process with varying parameters. Int. J. Multiphase Flow, 34: 869-878.

DOI: 10.1016/j.ijmultiphaseflow.2008.02.007

Jie, Y., L. Kun, F. Jianren, T. Yutaka and C. Kefa, 2008. Direct numerical simulation of particle dispersion in a turbulent jet considering inter-particle collisions. Int. J. Multiphase Flow, 34: 723-733. DOI: 10.1016/J.IJMULTIPHASEFLOW.2008.02.004

Jonas, J.B., I. Kreissig and R. Degenring, 2005. Intravitreal triamcinolone acetonide for treatment of intraocular proliferative, exudative and neovascular diseases. Prog. Retin. Eye Res., 24: 587-611. PMID: 16005407

Kosinski, P., A. Kosinska and A.C. Hoffmann, 2009. Simulation of solid particles behaviour in a driven cavity flow. Powder Technol., 191: 327-339. DOI: 10.1016/j.powtec.2008.10.025
Liao, J.I., 2002. Trajectory and velocity determination by smoother. PhD Thesis, National Taiwan University.

Matas, J.P., J.F. Morris and E. Guazzelli, 2004. Inertial migration of rigid spherical particles in Poiseuille flow. J. Fluid Mechan., 515: 171-195. DOI: 10.1017/S0022112004000254

Mussa, M., S. Abdullah, C.S.N. Azwadi and N. Muhamad, 2011. Simulation of natural convection heat transfer in an enclosure by the latticeBoltzmann method. Comput. Fluids, 44: 162-168. DOI: 10.1016/j.compfluid.2010.12.033

Saffman, P.G. and J.S. Turner, 1956. On the collision of drops in turbulent clouds. J. Fluid Mechan., 1: 1630. DOI: $10.1017 / \mathrm{S} 0022112056000020$

Shigeru, M. and S. Saito, 1970. On the mechanism of suspension of particles in horizontal pneumatic conveying: Monte Carlo simulation based on the irregular bouncing model. J. Chem. Eng. Japan, 3: 83-92.

Sidik, N.A.C. and M.R.N. Attarzadeh, 2012. The use of cubic interpolation method for transient hydrodynamics of solid particles. Int. J. Eng. Sci., 51: 90-103. DOI: 10.1016/j.ijengsci.2011.08.014

Sommerfeld, M., 2001. Validation of a stochastic Lagrangian modelling approach for inter-particle collisions in homogeneous isotropic turbulence. Int. J. Multiphase Flow, 27: 1829-1858. DOI: 10.1016/S0301-9322(01)00035-0

Tsorng, S.J., H. Capart, J.S. Lai and D.L. Young, 2006. Three-dimensional tracking of the long time trajectories of suspended particles in a lid-driven cavity flow. Exp. Fluids, 40: 314-328. DOI: 10.1007/s00348-005-0070-0

Ushijima, S. and N. Tanaka, 1996. Three-dimensional particle tracking velocimetry with laser-light sheet scannings. J. Fluids Eng., 118: 352-357.

Wolf-Gladrow, D.A., 2000. Lattice-Gas Cellular Automata and Lattice Boltzmann Models: An Introduction. 1st Edn., Springer, Berlin, ISBN-10: 3540669736, pp: 308.

Zhang, Y., R. Qin and D.R. Emerson, 2005. Lattice Boltzmann simulation of rarefied gas flows in microchannels. Phys. Rev. E., 71: 047702-047705. DOI: 10.1103/PhysRevE.71.047702 